\title{
The type III secretion system of Vibrio alginolyticus induces rapid apoptosis, cell rounding and osmotic lysis of fish cells
}

\author{
Correspondence \\ Chao-Qun $\mathrm{Hu}$ \\ cqhu@scsio.ac.cn
}

Received 16 April 2010

Revised 2 June 2010

Accepted 23 June 2010

\author{
Zhe Zhao, Chang Chen, Chao-Qun Hu, Chun-Hua Ren, Jing-Jing Zhao, \\ Lv-Ping Zhang, Xiao Jiang, Peng Luo and Oing-Bai Wang
}

\begin{abstract}
Key Laboratory of Applied Marine Biology of Guangdong Province (LAMB) and Key Laboratory of Marine Bio-resources Sustainable Utilization (LMB), South China Sea Institute of Oceanology, Chinese Academy of Sciences, Guangzhou 510301, PR China
\end{abstract}

\begin{abstract}
Vibrio alginolyticus is a Gram-negative bacterium and has been recognized as an opportunistic pathogen in humans as well as marine animals. However, the virulence mechanisms for this species of Vibrio have not been elucidated. This study characterized multiple mechanisms that induce cell death in fish cells upon infection with a $V$. alginolyticus strain, ZJO. The bacterium required its type III secretion system (T3SS) to cause rapid death of infected fish cells. Dying cells exhibited some features of apoptotic cells, such as membrane blebbing, nuclear condensation and DNA fragmentation. Further studies showed that caspase-3 was activated by the T3SS of the ZJO strain, confirming that infection with $V$. alginolyticus rapidly induces T3SS-dependent apoptosis in fish cells. Infection with the ZJO strain also led to membrane pore formation and release of cellular contents from infected fish cells, as evidenced by lactate dehydrogenase release and the uptake of a membrane-impermeable dye. Importantly, inhibition of apoptosis did not prevent ZJO-infected cells from releasing cellular contents and did not block cell rounding. Taken together, these data demonstrate that infection with $V$. alginolyticus may promote at least three different T3SS-dependent events, which lead to the death of fish cells. This study provides an important insight into the mechanism used by Vibrio species to cause host-cell death.
\end{abstract}

\section{INTRODUCTION}

Vibrio alginolyticus is a Gram-negative halophilic bacterium that is commonly found as part of the normal microbial flora in marine environments. It has been recognized as an opportunistic pathogen to both humans and marine animals (Balebona et al., 1998; Campanelli et al., 2008). V. alginolyticus can cause otitis, endophthalmitis and wound infections after direct contact with contaminated seawater or consumption of raw seafood. As global warming increases ocean temperatures, incidences of out-of-season infections caused by $V$. alginolyticus are escalating and drawing more attention (Sganga et al., 2009). Thus, there is a need to characterize the virulence mechanisms of $V$. alginolyticus.

The type III secretion system (T3SS) is a highly conserved apparatus among several Gram-negative bacteria; it delivers bacterial proteins, called effectors, directly into

Abbreviations: $\mathrm{AO}$, acridine orange; $\mathrm{EB}$, ethidium bromide; $\mathrm{EPC}$, Epithelioma papulosum cyprini; LDH, lactate dehydrogenase; STS, staurosporine; T3SS, type III secretion system; TUNEL, terminal dUTP nick-end labelling.

A supplementary figure and a supplementary table of primers are available with the online version of this paper. host cells across bacterial and host membranes (Hueck, 1998). Many of these effectors are virulence factors that can trigger host-cell death and manipulate the innate immune system (Coburn et al., 2007). Numerous other pathogenic bacteria, such as Yersinia spp., Pseudomonas aeruginosa, Salmonella spp. and Shigella spp., have been described to cause host-cell death using a T3SS apparatus (Dacheux et al., 2001; Fink \& Cookson, 2006; Monack et al., 1997; Nonaka et al., 2003). As effectors vary significantly between different bacterial systems, even within families of T3SSs, so do the mechanisms of cell death caused by them (Troisfontaines \& Cornelis, 2005). Apoptosis, pyroptosis, autophagy and oncosis are four distinct cell-death mechanisms triggered by bacteria; the dying cells display different morphological and physiological characteristics depending on the cell-death mechanism triggered (Fink \& Cookson, 2005).

Like most other Gram-negative pathogens, several Vibrio species, including Vibrio alginolyticus, Vibrio parahaemolyticus and Vibrio harveyi, also harbour gene clusters encoding a T3SS apparatus (Henke \& Bassler, 2004; Makino et al., 2003; Thompson et al., 2009). Moreover, two sets of T3SS gene clusters, T3SS1 and T3SS2, are present in a clinical strain of $V$. parahaemolyticus (Makino 
et al., 2003). In fact, T3SS1 is required for the cytotoxicity of $V$. parahaemolyticus in HeLa cells, whereas T3SS2 is involved in its enterotoxicity in rabbit ileal loops (Park et al., 2004). Interestingly, T3SS1 of $V$. parahaemolyticus may use more than one mechanism to induce the death of these same host-cell lines. For example, an early study showed that $V$. parahaemolyticus uses its T3SS1 to induce cell death in HeLa cells through apoptosis (Ono et al., 2006). Recently, Burdette et al. (2008) reported that $V$. parahaemolyticus T3SS1-mediated cytotoxicity is caspaseindependent and involves the induction of autophagy, followed by cell rounding and lysis. More recent studies also suggested that the bacterium kills host cells primarily via oncosis rather than apoptosis, pyroptosis or autophagy (Zhou et al., 2009). However, few reports are available regarding cell death caused by the T3SSs of other Vibrio species. Therefore, the mechanisms used by other Vibrio species to cause host-cell death are not well established.

In this study, we characterized $V$. alginolyticus-induced cell death in fish cell lines, and investigate the pathway of cell death. We confirmed that $V$. alginolyticus causes cell death in fish cells in a T3SS-dependent manner, and that the cell death occurs through multiple mechanisms, including induction of rapid apoptosis, cell rounding, and finally osmotic lysis.

\section{METHODS}

Bacterial strains, plasmids, and culture conditions. The bacterial strains and plasmids used are described in Table 1. Strain ZJO is the opaque/rugose variant of the wild-type $V$. alginolyticus strain ZJ51 (Chen et al., 2009) and was used as the parent strain for construction of deletion mutant $\Delta v s c C$. Escherichia coli strains S-17 and SY327 were used in gene deletion experiments. V. alginolyticus was routinely cultured in trypticase soy broth (TSB) or on $1.5 \%$ TSB agar plates (TSA) at $30{ }^{\circ} \mathrm{C}$. E. coli was cultured in Luria-Bertani (LB) medium at $37^{\circ} \mathrm{C}$. For selection of conjugates, thiosulfate-citrate-bile salts-sucrose agar (TCBS) was used with $10 \mu \mathrm{g}$ chloramphenicol $(\mathrm{Cm}) \mathrm{ml}^{-1}$. TSA and TSB with an additional $10 \%$ sucrose were used for the sucrose selection.

Construction of mutants and complementation strains. To construct the null mutant of the $v s c C$ gene in the ZJO strain, an inframe deletion was made by allelic exchange as described by Milton et al. (1996). Briefly, PCR was carried out to amplify the two fragments flanking the coding region of the $v s c C$ gene. The first fragment was amplified using primers $v s c C$-A, which incorporated a BglII site, and vscC-B (all primer sequences can be found in Supplementary Table S1 available with the online version of this paper), whereas primers $v s c C-\mathrm{C}$ and $v s c C$-D, incorporating a Sall site, were used to amplify the second fragment. Both fragments contained a $10 \mathrm{bp}$ overlapping sequence and were used as templates for the next PCR procedure, which used primers $v s c C$-A and $v s c C-\mathrm{D}$. The resulting fragment lacked all the coding sequence of the $v s c C$ gene and was inserted in the suicide vector $\mathrm{pDM} 4$, generating $\mathrm{pDM} 4 / \Delta v s c C$. The recombinant suicide plasmid was then transfected into $V$. alginolyticus strain ZJO. Mutant strain $\Delta v s c C$ was selected for on TCBS + Cm plates followed by a $10 \%$ sucrose selection process. Its presence was then confirmed by PCR and sequencing using primers $v s c C$-up and $v s c C$-down.

The complementation of the $\Delta v s c C$ mutant strain was performed as previously described (Zhao et al., 2009). Briefly, the ORF of the $v s c C$ gene and flanking DNA sequences was amplified using primers $v s c C$ $\mathrm{A}$ and $v s c C$-D. The fragment was ligated into pDM4, resulting in plasmid $\mathrm{pDM} 4 / v s c C$, which was introduced into the $\Delta v s c C$ mutant strain by conjugation. $\mathrm{Cm}$ - and Ampicillin (Ap)-resistant transconjugates were selected, and the bearing of the $v s c C$ gene was confirmed by PCR analysis and sequencing. The complemented mutant strain was named $\mathrm{C}-v s c C$.

Cell lines and infection. Epithelioma papulosum cyprini (EPC) cells (a representative carp fish cell line) were cultured in TC199 medium supplemented with $10 \%$ fetal bovine serum (FBS, Gibco) at $27{ }^{\circ} \mathrm{C} . \mathrm{V}$. alginolyticus was harvested from overnight cultures and pelleted by centrifugation at $10000 \mathrm{~g}$ for $2 \mathrm{~min}$ at $4{ }^{\circ} \mathrm{C}$. The bacterial pellets were resuspended in serum-free TC199 medium, and bacterial suspensions were added to the cell monolayer at an m.o.i. of 100 .

Table 1. Bacterial strains and plasmids used in this study

\begin{tabular}{|c|c|c|}
\hline \multicolumn{3}{|l|}{ E. coli } \\
\hline SY327 & $\Delta($ lac pro $) \arg E(\mathrm{Am})$ rif malA recA56 $\lambda$ pir lysogen & Miller \& Mekalanos (1988) \\
\hline BL21(DE3) & $\mathrm{F}^{-} o m p T h s d S_{B}\left(\mathrm{r}_{\mathrm{B}}^{-} \mathrm{m}_{\mathrm{B}}^{-}\right) g a l d c m(\mathrm{DE} 3)$ & Novagen \\
\hline \multicolumn{3}{|l|}{ V. alginolyticus } \\
\hline ZJO & $\begin{array}{l}\text { Opaque variant of wild strain ZJ51; isolated from diseased grouper fish off the } \\
\text { Southern China coast; } \mathrm{Ap}^{\mathrm{r}}\end{array}$ & Chen et al. (2009) \\
\hline$\Delta v s c C$ & ZJO carrying an in-frame deletion of $v s c C ; \mathrm{Ap}^{\mathrm{r}}$ & This study \\
\hline $\mathrm{C}-\nu s c \mathrm{C}$ & $\Delta v s c C$ complemented in trans with wild-type $v s c C$ gene; $\mathrm{Ap}^{\mathrm{r}} \mathrm{Cm}^{\mathrm{r}}$ & This study \\
\hline \multicolumn{3}{|l|}{ Plasmids } \\
\hline pDM4 & $\mathrm{Cm}^{\mathrm{r}}$; suicide vector with an R6K origin (pir-requiring) and sacBR of Bacillus subtilis & Milton et al. (1996) \\
\hline $\mathrm{pDM} 4 / \Delta v s c C$ & $\mathrm{Cm}^{\mathrm{r}} ; \mathrm{pDM} 4$ containing a mutant allele of $v s c C$ & This study \\
\hline $\mathrm{pDM} 4 / v s c C$ & $\mathrm{Cm}^{\mathrm{r}}$; pDM4 containing the wild-type allele of $v s c C$ & This study \\
\hline
\end{tabular}

${ }^{\star} \mathrm{Ap}^{\mathrm{r}}$, Ampicillin resistant; $\mathrm{Cm}^{\mathrm{r}}$, chloramphenicol resistant. 
Expression, purification, and antibody preparation of recombinant Va1680. The coding region corresponding to the Val680 gene of the ZJO strain was cloned into pET-32a $(+)$ vector (Novagen) using primers val680-F and val680-R (Supplementary Table S1) to generate the recombinant plasmid pET32a/va1680. The construct was transformed into E. coli BL21(DE3), and the bacteria were induced for $6 \mathrm{~h}$ with $0.5 \mathrm{mM}$ IPTG at $30{ }^{\circ} \mathrm{C}$ to express the recombinant Va1680 protein. Purification and antibody preparation of the recombinant Va1680 were then performed as described previously (Zhao et al., 2008).

Immunofluorescent staining. To confirm that the $\Delta v s c C$ mutant strain T3SS was dysfunctional in protein secretion, immunofluorescent staining was carried out using the Va1680 antibody. EPC cells were infected for $2 \mathrm{~h}$ with the ZJO strain, $\Delta v s c C$ or C- $v s c C$, then fixed with $4 \%$ paraformaldehyde in PBS for $20 \mathrm{~min}$, and permeabilized with $0.1 \%$ Triton X-100 in PBS for $15 \mathrm{~min}$ at room temperature. After blocking in $10 \%$ normal goat serum at room temperature for $1 \mathrm{~h}$, the cells were incubated with Va1680 antibody in $1 \%$ normal goat serum for $2 \mathrm{~h}$, rinsed three times for 10 min each with PBS containing $1 \%$ normal goat serum, and incubated with Alexa Fluor 488 goat anti-mouse IgG $(\mathrm{H}+\mathrm{L})$ (Molecular Probes). All samples were examined under a Leica DM IRB fluorescence microscope.

Hoechst staining. EPC cells infected with wild-type ZJO, $\Delta v s c C$ or C- $v s c C$, were stained with Hoechst 33258 (Sigma) at indicated time points post-infection as described by Burdette et al. (2008). Cells were left untreated or were treated with $2 \mu \mathrm{M}$ staurosporine (STS) as controls. The fluorescence signal was detected under a Leica DM IRB fluorescence microscope.

Lactate dehydrogenase (LDH) release assay. EPC cells were infected with strains of $V$. alginolyticus, as described, over a $3 \mathrm{~h}$ timecourse. Cells were left untreated or were treated with $2 \mu \mathrm{M}$ STS as controls. LDH release was measured with the Cytotoxicity Detection kit $^{\text {PLUS }}$ according to the manufacturer's instructions (Roche). Results are expressed as LDH release calculated as a percentage of total cell lysis by the lysis buffer provided in the kit.

Scanning electron microscopy. EPC cells were infected or treated as indicated above for $2 \mathrm{~h}$, fixed in $2 \%$ glutaraldehyde (Sigma), treated with osmium tetroxide in sodium cacodylate buffer, dehydrated with sequential washes in $30 \%, 50 \%, 70 \%, 80 \%, 90 \%$ and $100 \%$ ethanol, and then dried using critical-point drying before coating with gold. The samples were examined by scanning electron microscopy (HITACHI S-3400).

In situ detection of fragmented DNA. DNA fragmentation was detected by the terminal dUTP nick-end labelling (TUNEL) method using the In situ Cell Death Detection kit, Fluorescein (Roche). Briefly, EPC cells were seeded on sterile glass coverslips in six-well plates overnight to achieve a monolayer and then infected with strains of $V$. alginolyticus for $2 \mathrm{~h}$. The infected cells were rinsed once with PBS and fixed with $4 \%$ paraformaldehyde in PBS for $1 \mathrm{~h}$. After fixation, the coverslips were incubated in permeabilization solution $(0.1 \%$ Triton X-100 in $0.1 \%$ sodium citrate) for $2 \mathrm{~min}$ on ice and labelled using the TUNEL reaction mixture. EPC cells were treated with $2 \mu \mathrm{M}$ STS for $2 \mathrm{~h}$ for the positive control, or left untreated for the negative control. All samples were analysed under a Leica DM IRB fluorescence microscope.

Colorimetric assays for caspase-1/-3 activity in infected cells. Measurements of caspase-1 and caspase-3 activity during $V$. alginolyticus infection were performed using commercially available caspase- 1 and caspase- 3 assay kits (BioVision). EPC cells were left uninfected, infected with $V$. alginolyticus strains as described, or treated with $2 \mu \mathrm{M}$ STS, and then lysed on ice for $10 \mathrm{~min}$ with the cell lysis buffer provided in the kit at the indicated time points. After centrifugation, $50 \mu \mathrm{l}$ supernatant (about $120 \mu \mathrm{g}$ protein) was mixed with $50 \mu \mathrm{l} 2 \times$ reaction buffer, and $5 \mu \mathrm{l} 4 \mathrm{mM}$ YVAD-pNA or DEVD$p \mathrm{NA}$ substrate for measuring caspase-1 or caspase-3 activity, respectively. The reaction mixtures were incubated at $37^{\circ} \mathrm{C}$ for $2 \mathrm{~h}$, and levels of cleaved caspase substrate were measured at $405 \mathrm{~nm}$ in a microplate spectrophotometer (Thermo Labsystems Ascent). The data were expressed as the fold increase of caspase activity compared to the corresponding values in lysates of uninfected cells.

Caspase inhibitor assay. For the caspase inhibitor assay, EPC cells were incubated with $100 \mu \mathrm{M}$ of the general caspase inhibitor Z-VADFMK, the caspase-1 inhibitor Z-WEHD-FMK or the caspase-3 inhibitor Z-DEVD-FMK (R\&D Systems) for $1 \mathrm{~h}$ before infection with $V$. alginolyticus or treatment with $2 \mu \mathrm{M}$ STS. DNA fragmentation and LDH release were assayed to evaluate the inhibitory effect of the three caspase inhibitors after $2 \mathrm{~h}$ of infection or treatment using the In situ Cell Death Detection kit and Cytotoxicity Detection kit ${ }^{\text {PLUS }}$ (Roche) as described above.

Acridine orange and ethidium bromide double staining. To detect pore formation in the host-cell membrane upon infection with $V$. alginolyticus strains, EPC cells were stained at $1 \mathrm{~h}$ post-infection with freshly prepared acridine orange/ethidium bromide $(\mathrm{AO} / \mathrm{EB})$ dye mix $\left(10 \mu \mathrm{g} \mathrm{ml}^{-1} \mathrm{AO}\right.$ and $10 \mu \mathrm{g} \mathrm{ml}^{-1} \mathrm{~EB}$ in PBS) for $30 \mathrm{~min}$, followed by fixation with $4 \%$ paraformaldehyde for $15 \mathrm{~min}$ in the dark. After washing with PBS, cell morphology was observed with a Leica DM IRB fluorescence microscope.

Osmoprotection experiments. Osmoprotection experiments were carried out as described previously (Fink \& Cookson, 2006; Zhou et al., 2009). Briefly, EPC cells were infected with the ZJO strain or treated with $10 \mathrm{mM} \mathrm{H} \mathrm{H}_{2} \mathrm{O}_{2}$ in the presence of $30 \mathrm{mM}$ solutions of sucrose (Amresco), PEG600, PEG1000, PEG1450 or PEG 3350 (Sigma) made up in serum-free TC199 medium and filter-sterilized prior to use. LDH release was measured to evaluate the protection effect after $2 \mathrm{~h}$ of infection using a Cytotoxicity Detection kit ${ }^{\text {PLUS }}$ (Roche) as described.

Statistical analysis. Statistical analysis was performed with one-way analysis of variance (ANOVA) and $P$-values $<0.05$ were considered statistically significant. Pairwise comparisons were conducted using a Tukey's multiple comparison test and the analyses were conducted using the SPSS software (version 15.0).

\section{RESULTS}

\section{The $\Delta v s c C$ mutant strain T3SS is dysfunctional in protein secretion}

To examine the role of the T3SS of $V$. alginolyticus in induction of cell death, we constructed a ZJO mutant strain $(\Delta v s c C)$ lacking the $v s c C$ gene, which encodes an outermembrane protein of the T3SS apparatus (Park et al., 2004). To confirm that the mutant strain was defective in T3SS protein secretion, immunofluorescent staining was carried out using antibodies against Va1680 of the ZJO strain. Va1680 is an orthologue of Vp1680 (VopQ) from $V$. parahaemolyticus, which is an effector protein secreted by the T3SS (Burdette et al., 2009; Ono et al., 2006). As shown in Fig. 1, the ZJO-infected cells were detected with the Va1680 antibody (Fig. 1d), whereas the $\Delta v s c C$-infected cells were not. However, the $\Delta v s c C$ mutant strain itself was labelled by 


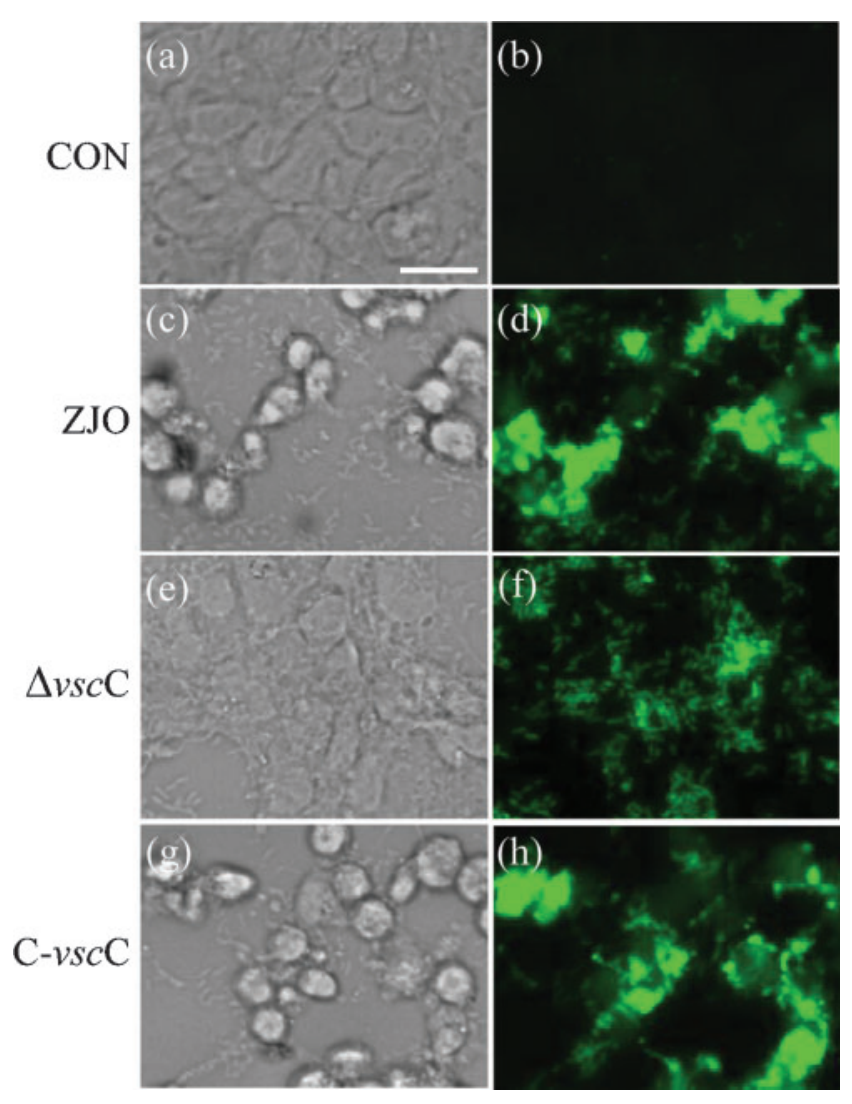

Fig. 1. Secretion assay of the T3SS in $V$. alginolyticus detected by immunofluorescent staining. EPC cells were infected for $2 \mathrm{~h}$ with strain ZJO (c, d), $\Delta v s c C$ (e, f), or C-vscC ( $g, h$ ) as described. Cells were then fixed, permeabilized and stained with the Va1680 antibody and Alexa Fluor 488-conjugated anti-mouse antibody. Uninfected cells $(a, b)$ were used as a negative control. Scale bar, $50 \mu \mathrm{m}$.

antibodies against Va1680 (Fig. 1f). These data revealed that the $\Delta v s c C$ could not translocate the Va1680 protein into EPC cells via the T3SS apparatus during infection, confirming that the mutant has a T3SS that is dysfunctional in protein secretion. Furthermore, the complemented strain restored the wild-type T3SS-dependent secretion phenotype that was lacking in $\Delta v s c C$ (Fig. 1g, h), indicating that the mutagenesis is specific and does not introduce other significant changes into the $v s c C$ deletion strain.

\section{The T3SS is required for $V$. alginolyticus-induced cell death}

As shown in Fig. 1(c, g), almost all EPC cells infected with the $\mathrm{ZJO}$ or the $\mathrm{C}-v s c C$ strains for $2 \mathrm{~h}$ exhibited rounding, and many of them appeared to undergo lysis, whereas EPC cells infected with the $\Delta v s c C$ T3SS mutant strain for $2 \mathrm{~h}$ maintained a uniform monolayer and normal cell appearance similar to that of uninfected cells (Fig. 1a,e), demonstrating that the T3SS is required for $V$. alginolyticus-induced cell death. To further characterize the mechanism of cell death caused by the T3SS of $V$. alginolyticus, nuclear morphology was visualized by staining with Hoechst 33258. Nuclear condensation was observed in some ZJOor C-vscC-infected cells at $30 \mathrm{~min}$ post-infection (Fig. $2 \mathrm{~d}, \mathrm{j}$ ), and as the infection time increased, the number of cells undergoing nuclear fragmentation and the formation of apoptotic bodies also increased (Fig. 2e-f, k-l). This phenotype resembles that seen in cells treated with STS, a strong inducer of apoptosis (Fig. $2 \mathrm{~m}-\mathrm{O}$ ). In contrast, the nuclear compartments in $\Delta v s c C$-infected cells remained intact during the course of infection, which was consistent with the morphology of the uninfected cells (Fig. 2a-c, g-i). Taken together, these data suggest that $V$. alginolyticus infection rapidly induces cell death in a T3SS-dependent manner, and that dead cells display cell rounding, nuclear condensation and fragmentation.

\section{V. alginolyticus T3SS-induced cell death displays characteristics of apoptosis}

Infected cells were also examined by scanning electron microscopy to further analyse the morphological changes after infection with $V$. alginolyticus. At $2.5 \mathrm{~h}$ post-infection or treatment, several EPC cells exposed to the ZJO strain, as well as those treated with STS, displayed obvious membrane blebbing, a characteristic of apoptosis (Fig. 3a). By contrast, EPC cells infected with the $\Delta v s c C$ mutant strain did not display membrane blebbing, although the disappearance of filopodia was apparent (Fig. 3a). DNA fragmentation is one of the typical characteristics of apoptotic cells and can be detected with the TUNEL assay (Kressel \& Groscurth, 1994). The results in Fig. 3(b, c) show that when the wild-type ZJO strain was used to infect EPC cells, a mean of $73 \%$ of cells were TUNEL positive at $2 \mathrm{~h}$ post-infection. In contrast, the TUNEL signal in uninfected cells and cells infected with the $\Delta v s c C$ mutant strain was almost undetectable (Fig. 3b, c). STS-infected EPC cells were the positive control for apoptosis and $14 \%$ of cells were TUNEL positive (Fig. 3b, c). Based on these observations, it can be inferred that $V$. alginolyticus T3SSinduced cell death exhibits characteristics of apoptotic death similar to those induced by STS treatment.

\section{V. alginolyticus infection rapidly induces T3SS-dependent apoptosis}

Pyroptosis is a more recently identified pathway of eukaryotic cell death, and pyroptotic cells also undergo nuclear condensation and DNA fragmentation like apoptotic cells (Labbe \& Saleh, 2008). To test whether $V$. alginolyticus infection induces apoptosis or pyroptosis, we assayed the activation of caspase- 1 and caspase- 3 in infected cells. As shown in Fig. 4(a), caspase-3 in the ZJO-infected cells was obviously synthesized after $1 \mathrm{~h}$ of infection and reached levels approximately 10-fold higher than those induced by STS. In contrast, caspase- 1 activity was not detected in the ZJO-infected and STS-treated cells over the 


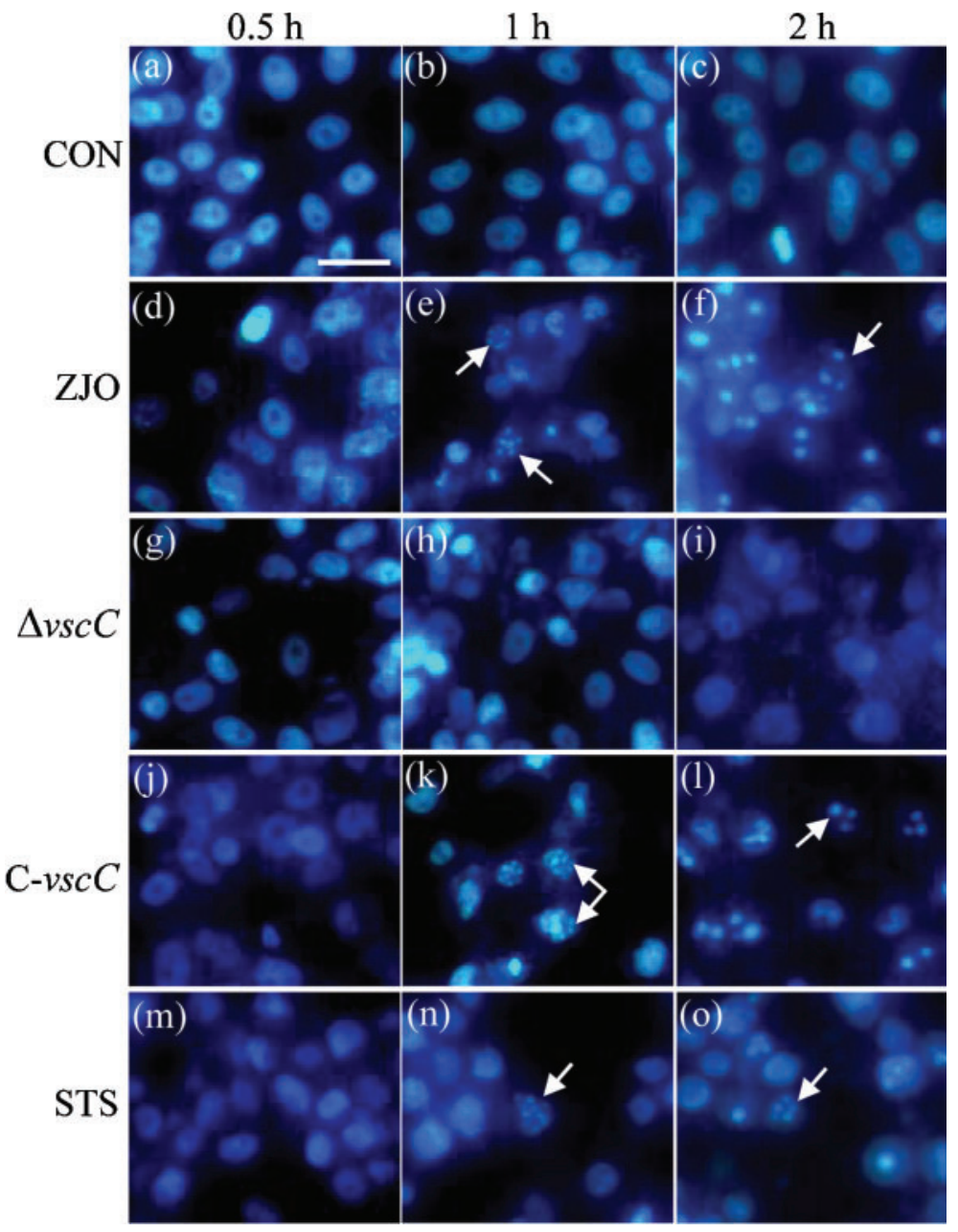

Fig. 2. V. alginolyticus infection of EPC cells induces nuclear condensation and fragmentation in a T3SS-dependent manner. EPC cells were infected with strain ZJO (d-f), $\Delta v s c C$ $(\mathrm{g}-\mathrm{i})$ or $\mathrm{C}-v s c \mathrm{C}(\mathrm{j}-\mathrm{l})$ as described. Cells were treated with $2 \mu \mathrm{M}$ STS for the positive control $(\mathrm{m}-\mathrm{o})$ or left uninfected $(\mathrm{a}-\mathrm{c})$ for the negative control. Nuclear condensation and fragmentation were visualized using Hoechst 33258 to stain nuclei (blue) at indicated time points after infection. Arrows indicate the fragmented nucleus. Scale bar, $50 \mu \mathrm{m}$.

same period of time (Fig. 4b). To further confirm that caspase-3 activity is involved in $V$. alginolyticus-induced cell death, we treated EPC cells with three caspase inhibitors for $1 \mathrm{~h}$ prior to infection and measured DNA fragmentation with the TUNEL assay. Results showed that both the general caspase inhibitor and the more specific caspase-3 inhibitor almost completely prevented the ZJO-infected cells from undergoing DNA fragmentation, whereas the caspase-1 inhibitor did not block DNA fragmentation in the ZJOtreated cells (Fig. 4c). Therefore, caspase-3 is activated during $V$. alginolyticus infection and is involved in T3SSinduced DNA fragmentation. Thus, V. alginolyticus infection triggers an apoptotic pathway involving caspase- 3 in a T3SS-dependent manner.

\section{LDH release mediated by the T3SS of $\boldsymbol{V}$. alginolyticus is independent of caspase activity}

Apoptosis is a noninflammatory type of cell death in which dying cells do not release their cytoplasmic contents into the extracellular space (Fink \& Cookson, 2005). Unexpectedly, we observed a significant $\mathrm{LDH}$ release into the medium as early as $30 \mathrm{~min}$ after infection with the ZJO strain. As the infection time increased, LDH concentrations in the medium also increased, reaching more than $80 \%$ total $\mathrm{LDH}$ release at $1.5 \mathrm{~h}$ post-infection (Fig. 5a). Conversely, LDH levels in the medium of cells infected with the $\Delta v s c C$ mutant or treated with STS were not significantly different from those in the medium of uninfected cells (Fig. 5a). More importantly, analysis of $\mathrm{LDH}$ release in the presence of caspase inhibitors revealed that three different inhibitors did not reduce the level of LDH that was released into the medium (Fig. 5b) and did not block cell rounding (data not shown). This indicates that caspase activity was not involved in LDH release and cell rounding. Additionally, the general caspase and caspase-3 inhibitors slightly restrained LDH release from STS-treated EPC cells, although the inhibitory effect was not significant (Fig. 5b). Therefore, we hypothesize that $V$. alginolyticus uses its T3SS to initiate the release of cellular contents and to induce cell rounding in infected EPC cells, independent of apoptotic pathway induction.

\section{The $V$. alginolyticus T3SS induces pore formation leading to osmotic lysis}

It has been suggested that many bacteria use their T3SS to create pores in host cell membranes directly, thereby 
(a)

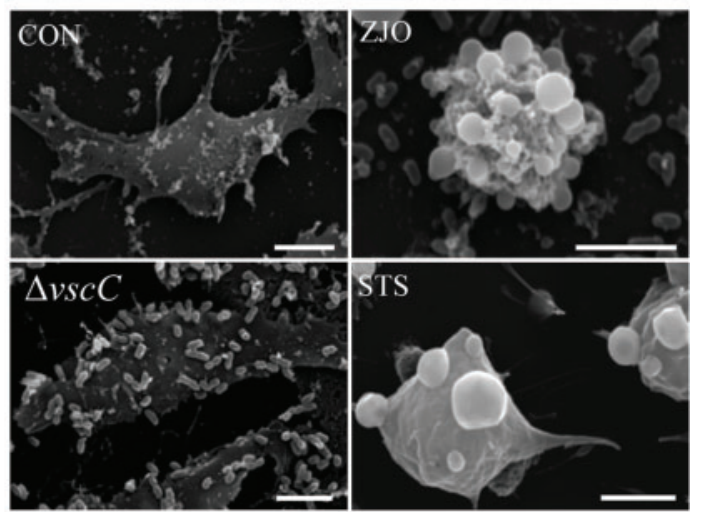

(b)

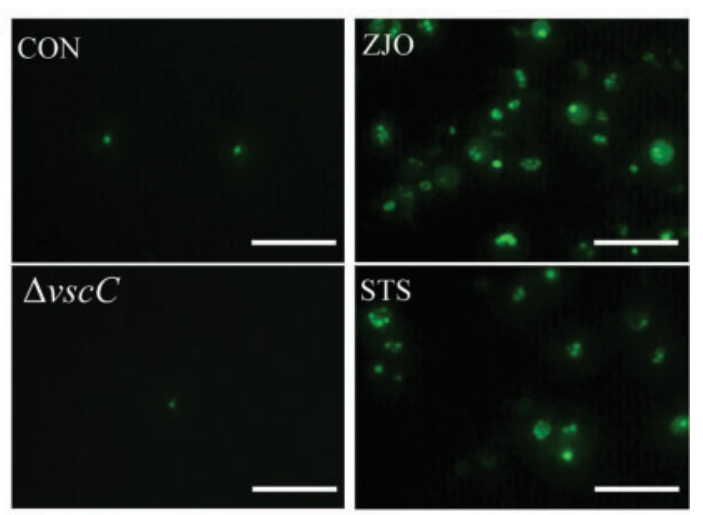

(c)

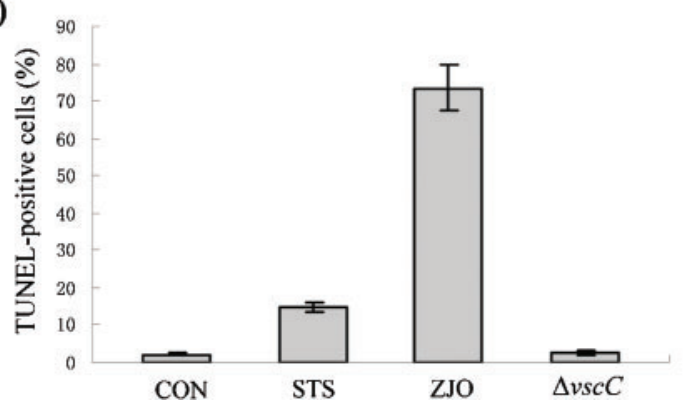

Fig. 3. V. alginolyticus infection of EPC cells induces membrane blebbing and DNA fragmentation in a T3SS-dependent manner. (a) Scanning electron micrographs of EPC cells either infected with strain ZJO or $\Delta v s c C$, treated with $2 \mu \mathrm{M}$ STS, or left without treatment $(\mathrm{CON})$ for $2.5 \mathrm{~h}$. Scale bars, $5 \mu \mathrm{m}$. (b) The detection of DNA fragmentation by TUNEL staining, under fluorescence microscopy. EPC cells were either uninfected, infected with wild-type ZJO and the $\Delta v s c C$ mutant strain, or treated with $2 \mu \mathrm{M}$ STS for $2 \mathrm{~h}$. Green fluorescence indicates a positive TUNEL signal and the resulting DNA fragmentation. Scale bars, $100 \mu \mathrm{m}$. (c) TUNEL-positive cells were quantified from three independent experiments and the data are presented as means \pm SE. A minimum of 600 cells were examined for each experiment.

causing osmotic lysis (Dacheux et al., 2001; Fink \& Cookson, 2006; Neyt \& Cornelis, 1999; Nonaka et al., 2003; Zhou et al., 2009). Based on the above observations, we hypothesized that LDH release caused by V. alginolyticus (a)

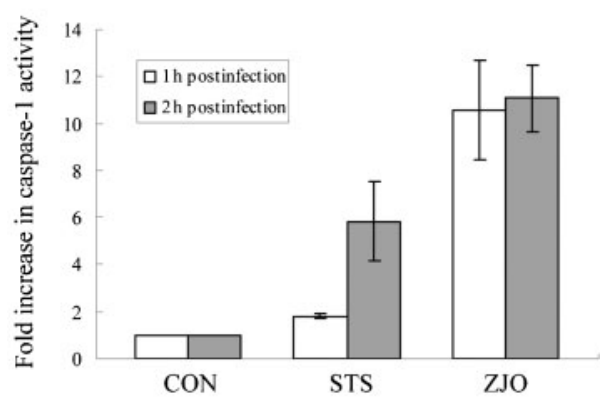

(b)

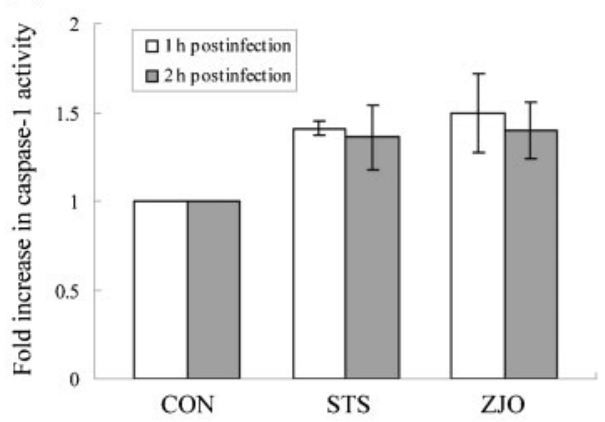

(c)

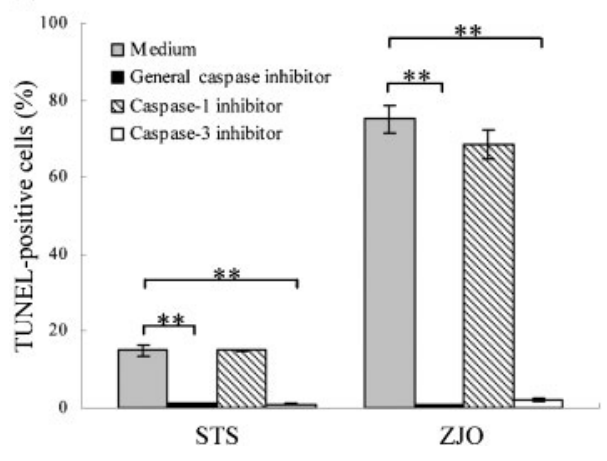

Fig. 4. V. alginolyticus infection of EPC cells activates a T3SSdependent apoptotic pathway. $(a, b)$ Measurement of caspase-3 and caspase- 1 activity. EPC cells that were either uninfected, infected with wild-type ZJO, or treated with $2 \mu \mathrm{M}$ STS, were collected at indicated time points after infection and the levels of cleaved caspase substrate were measured. The data are expressed as fold-increase compared to the corresponding values of caspase activity in uninfected cells. (c) Inhibitory effect of three caspase inhibitors on $V$. alginolyticus-induced DNA fragmentation. EPC cells were pre-incubated for $1 \mathrm{~h}$ with $100 \mu \mathrm{M}$ of one of three caspase inhibitors and then infected with ZJO or treated with $2 \mu \mathrm{M}$ STS for $2 \mathrm{~h}$. Inhibitory effects were evaluated using the TUNEL assay. The data are expressed as means \pm SE from three independent experiments. ${ }^{\star *}, P<0.01$ by one-way ANOVA and Tukey's multiple-comparison test.

is a result of osmotic lysis. To test this hypothesis, pore formation was analysed by $\mathrm{AO} / \mathrm{EB}$ double staining. $\mathrm{AO}$ is a membrane-permeable dye that stains both live and dead cells, and exhibits green fluorescence, whereas EB is a 
(a)

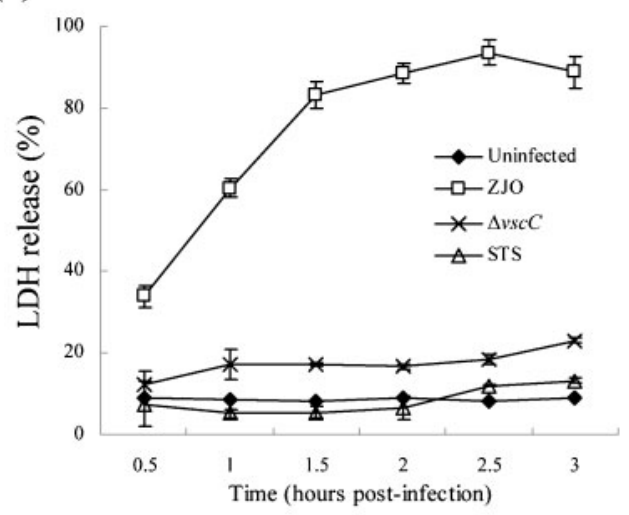

(b)

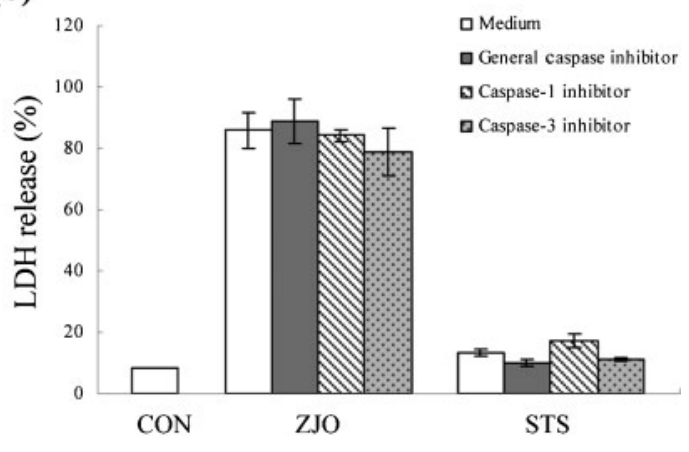

Fig. 5. LDH release mediated by the T3SS of $V$. alginolyticus is independent of caspase activity. (a) LDH release caused by $V$. alginolyticus infection. EPC cells were infected with the ZJO strain or the $\Delta v s c C$ mutant, or treated with $2 \mu \mathrm{M}$ STS over a $3 \mathrm{~h}$ time period. Cells were left uninfected as the negative control. LDH release was calculated as a percentage of total lysis of cells by the lysis buffer provided in the kit. (b) Inhibitory effect of three caspase inhibitors on LDH release. EPC cells were pre-incubated for $1 \mathrm{~h}$ with $100 \mu \mathrm{M}$ each of three caspase inhibitors and then infected with the ZJO strain or treated with $2 \mu \mathrm{M}$ STS for $2 \mathrm{~h}$. Inhibitory effects were evaluated using LDH release assays. The data are expressed as means \pm SE from three independent experiments.

membrane-impermeable dye that stains only cells that have lost membrane integrity. When cells with permeable membranes are double stained by $\mathrm{AO}$ and $\mathrm{EB}$, they display yellow or orange-yellow fluorescence, which confirms membrane permeability. Our results showed that EPC cells infected with the ZJO strain exhibited an orange-yellow background under fluorescence microscopy, and cells treated with STS displayed a yellow-green fluorescence (Fig. 6a). In contrast, uninfected cells and cells infected with the $\Delta v s c C$ mutant strain were uniformly green in colour (Fig. 6a). Thus, V. alginolyticus infection causes a T3SSdependent pore formation in the cell membrane of fish cells.

Osmotic lysis resulting from membrane pores can be prevented by osmoprotectant molecules with molecular diameters greater than the functional diameter of the pores (Fink \& Cookson, 2006). To further determine whether $V$. (a)

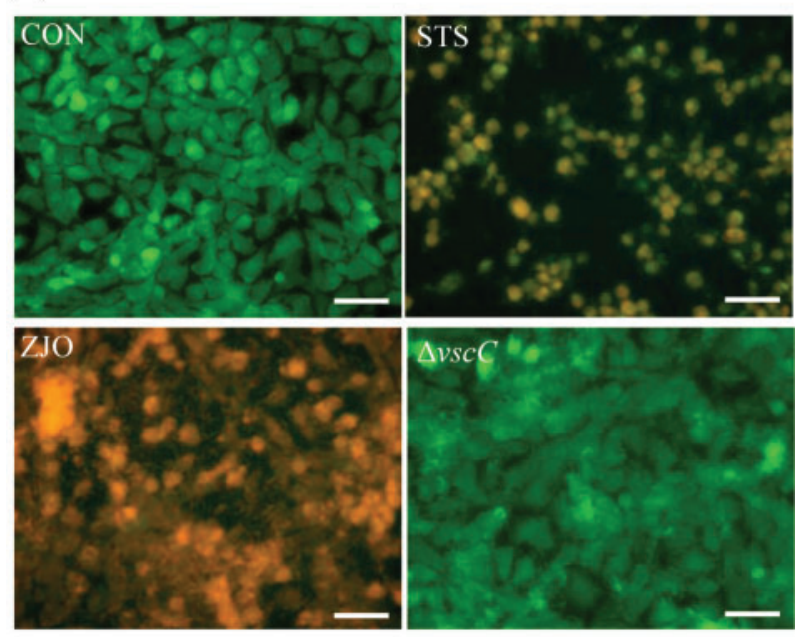

(b)

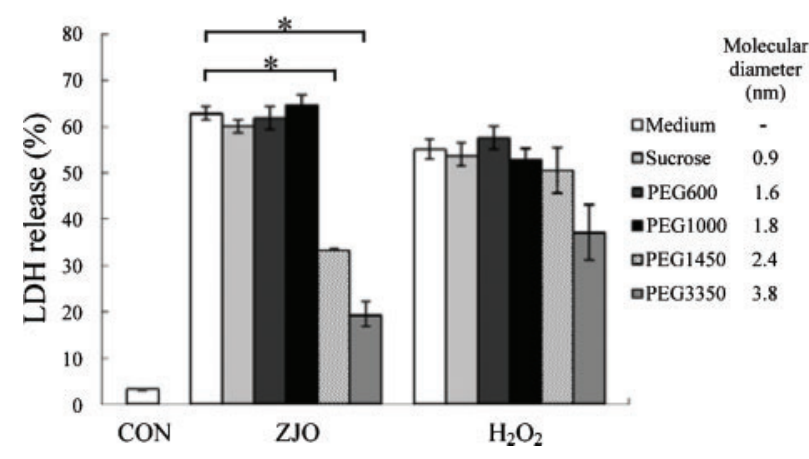

Fig. 6. Membrane pore formation of EPC cells by the T3SS of $V$. alginolyticus. (a) AO/EB double staining. EPC cells were uninfected, infected with strain ZJO or $\Delta v s c C$, or treated with $2 \mu \mathrm{M}$ STS for $2 \mathrm{~h}$, and then were stained with the membranepermeable dye $\mathrm{AO}$ and the membrane-impermeable dye EB as described. Cells were visualized fluorescence microscopy. Scale bars, $100 \mu \mathrm{m}$. (b) Osmoprotectant assay. LDH release was quantified from EPC cells infected with the ZJO strain or treated with $10 \mathrm{mM} \mathrm{H}_{2} \mathrm{O}_{2}$ in the presence of osmoprotectants of varying sizes. The data are expressed as means $\pm S E$ from three independent experiments. ${ }^{*}, P<0.05$ by one-way ANOVA and Tukey's multiple-comparison test.

alginolyticus T3SS-induced pore formation is responsible for osmotic lysis, osmoprotection experiments were performed. LDH release in ZJO-infected cells was significantly decreased in the presence of osmoprotectant molecules that were $2.4 \mathrm{~nm}$ in diameter or larger, whereas those smaller than $1.8 \mathrm{~nm}$ had no effect, indicating that the poresize caused by the T3SS of $V$. alginolyticus is between $1.8 \mathrm{~nm}$ and $2.4 \mathrm{~nm}$ (Fig. 6b). None of the osmoprotectant molecules prevented lysis of $\mathrm{H}_{2} \mathrm{O}_{2}$-treated controls (Fig. 6b). Collectively, these findings indicate that $V$. alginolyticus infection causes the formation of membrane 
pores in a T3SS-dependent manner and thereby results in osmotic lysis of infected cells.

\section{DISCUSSION}

Cell death is the most common outcome of host-bacterial pathogen interactions and has important consequences for pathogenesis (Labbe \& Saleh, 2008). Apoptosis is a wellknown mechanism of programmed cell death and is mechanistically mediated by caspases, which are cysteinedependent aspartate-specific proteases. Active caspases cleave cellular substrates, resulting in the characteristic features of apoptosis, which include membrane blebbing, nuclear condensation, DNA fragmentation and maintenance of an intact plasma membrane (Samali et al., 1999; Elmore, 2007). Upon infection by $V$. alginolyticus, we observed the T3SS-mediated appearance of typical apoptotic features in EPC cells, such as cell rounding, DNA fragmentation, formation of apoptotic bodies and membrane blebbing (Figs 1-3). However, these characteristics alone are not sufficient to prove that apoptosis has occurred, because cells induced to undergo the pyroptosis pathway show similar properties (Fink \& Cookson, 2005). Therefore, we examined events that are unique to apoptosis, such as activation of apoptotic caspases and poly (ADP-ribose) polymerase (PARP) cleavage. Caspase-3 plays a key role in the execution of apoptosis and is responsible for PARP cleavage and DNA fragmentation, whereas caspase- 1 activation is a defining feature of pyroptosis and is not involved in apoptosis (Labbe \& Saleh, 2008). Our data demonstrate that $V$. alginolyticus infection activated caspase- 3 and not caspase- 1 in EPC cells (Fig. 4), confirming that $V$. alginolyticus infection activates an apoptotic pathway that is T3SS dependent.

As described above, apoptotic cells maintain plasma membrane integrity and do not release their cellular contents (Elmore, 2007). However, LDH release was detected from $V$. alginolyticus-infected EPC cells when the apoptotic pathway was activated (Fig. 5a). Subsequently, we verified that membrane pores dependent on the T3SS of $V$. alginolyticus were responsible for the release of cellular contents (Fig. 6), as it has been shown that membrane defects can cause osmotic lysis by dissipating cellular ionic gradients (Fink \& Cookson, 2006). Importantly, caspase inhibition did not prevent the release of cellular contents from $V$. alginolyticusinfected cells and did not block cell rounding (Fig. 5b). These findings suggest that the apoptotic pathway is not involved in cell rounding and osmotic lysis during infection by $V$. alginolyticus, although they are all mediated by the T3SS apparatus. In addition, to avoid the bias of EPC cells, we characterized the cell-death phenotype in a fish mononuclear cell line (carp leukocyte cell, CLC) following infection with $V$. alginolyticus. Data from the infection of CLC cells revealed that the mechanism of cell death was consistent with that observed in EPC cells (unpublished data). Therefore, we propose that $V$. alginolyticus uses its T3SS to evoke at least three different events - apoptosis, cell rounding and osmotic lysis - which lead to the cell death of fish cells within hours of infection.

Understanding why $V$. alginolyticus causes cell death at such an accelerated rate by using the three different events during infection will require more research; however, one possibility is that $V$. alginolyticus may initially take advantage of apoptosis to degrade intracellular organelles to provide readily usable nutrients, such as nucleotides and amino acids. After cell lysis, the bacteria rapidly gain access to those essential nutrients to survive and propagate before being expelled from the host. $V$. alginolyticus is the most prevalent species of Vibrio in the marine environment and usually lives in oligotrophic conditions (Dawson et al., 1981; Xie et al., 2005). As an opportunistic pathogen, it may utilize the above mechanism to quickly establish environmental niches in a variety of host tissues and to penetrate host barrier defences.

$V$. alginolyticus is closely related to $V$. parahaemolyticus and $V$. harveyi (Thompson et al., 2009). Recently, we determined the genomic sequence of the T3SS of $V$. alginolyticus ZJO strain (GenBank accession no. GU074526). Sequence analysis revealed that the island was similar to T3SS1 of $V$. parahaemolyticus in synteny and the identity of each predicted protein (Makino et al., 2003; Park et al., 2004). Interestingly, $V$. alginolyticus induces apoptosis during the process of T3SS-mediated cell death, whereas $V$. parahaemolyticus does not. In contrast to our findings, the T3SS1-mediated cell death of $V$. parahaemolyticus preserves the integrity of the host-cell nuclear membrane and does not activate caspase molecules (Burdette et al. 2008; Zhou et al., 2009). This difference may be due to the inherent differences in the T3SS effectors of $V$. alginolyticus and $V$. parahaemolyticus, as there were some minor differences between the hypothetical regions encoding them (Supplementary Fig. S1). Therefore, future studies should characterize the key effectors secreted by the $V$. alginolyticus T3SS that are responsible for apoptosis activation and clarify their interactions with cellular proteins.

In summary, we describe here multiple mechanisms used by the T3SS of $V$. alginolyticus to induce cell death in fish cells, whereby the bacterial infection rapidly induces apoptosis and causes cell rounding and osmotic lysis. Furthermore, inhibition of apoptosis does not prevent cell rounding and the eventual death of the infected cell. The death process is different from that reported for $V$. parahaemolyticus and will provide novel insight into the mechanisms used by Vibrio species to induce host-cell death.

\section{ACKNOWLEDGEMENTS}

We thank Dr Fansuo Geng (University of Sheffield) and Dr Bryan Danielson (Baylor College of Medicine) for English language editing, and Dr Liu Hong for providing the fish cell lines. The authors also 
gratefully acknowledge the support of the K. C. Wong Education Foundation, Hong Kong. This research was supported by the National Basic Research Program of China (2006CB101803) and in part by the National Natural Science Foundation of China (30901117) and the China Postdoctoral Science Foundation (20090450100).

\section{REFERENCES}

Balebona, M. C., Andreu, M. J., Bordas, M. A., Zorrilla, I., Moriñigo, M. A. \& Borrego, J. J. (1998). Pathogenicity of Vibrio alginolyticus for cultured gilt-head sea bream (Sparus aurata L.). Appl Environ Microbiol 64, 4269-4275.

Burdette, D. L., Yarbrough, M. L., Orvedahl, A., Gilpin, C. J. \& Orth, K. (2008). Vibrio parahaemolyticus orchestrates a multifaceted host cell infection by induction of autophagy, cell rounding, and then cell lysis. Proc Natl Acad Sci U S A 105, 12497-12502.

Burdette, D. L., Seemann, J. \& Orth, K. (2009). Vibrio VopQ induces PI3-kinase-independent autophagy and antagonizes phagocytosis. Mol Microbiol 73, 639-649.

Campanelli, A., Sanchez-Politta, S. \& Saurat, J. H. (2008). Cutaneous ulceration after an octopus bite: infection due to Vibrio alginolyticus, an emerging pathogen. Ann Dermatol Venereol 135, 225-227.

Chen, C., Xie, J. \& Hu, C. Q. (2009). Phenotypic and genetic differences between opaque and translucent colonies of Vibrio alginolyticus. Biofouling 25, 525-531.

Coburn, B., Sekirov, I. \& Finlay, B. B. (2007). Type III secretion systems and disease. Clin Microbiol Rev 20, 535-549.

Dacheux, D., Goure, J., Chabert, J., Usson, Y. \& Attree, I. (2001). Pore-forming activity of type III system-secreted proteins leads to oncosis of Pseudomonas aeruginosa-infected macrophages. Mol Microbiol 40, 76-85.

Dawson, M. P., Humphrey, B. A. \& Marshall, K. C. (1981). Adhesion: a tactic in the survival strategy of a marine vibrio during starvation. Curr Microbiol 6, 195-199.

Elmore, S. (2007). Apoptosis: a review of programmed cell death. Toxicol Pathol 35, 495-516.

Fink, S. L. \& Cookson, B. T. (2005). Apoptosis, pyroptosis, and necrosis: mechanistic description of dead and dying eukaryotic cells. Infect Immun 73, 1907-1916.

Fink, S. L. \& Cookson, B. T. (2006). Caspase-1-dependent pore formation during pyroptosis leads to osmotic lysis of infected host macrophages. Cell Microbiol 8, 1812-1825.

Henke, J. M. \& Bassler, B. L. (2004). Quorum sensing regulates Type III secretion in Vibrio harveyi and Vibrio parahaemolyticus. J Bacteriol 186, 3794-3805.

Hueck, C. J. (1998). Type III protein secretion systems in bacterial pathogens of animals and plants. Microbiol Mol Biol Rev 62, 379-433.

Kressel, M. \& Groscurth, P. (1994). Distinction of apoptotic and necrotic cell death by in situ labelling of fragmented DNA. Cell Tissue Res 278, 549-556.

Labbe, K. \& Saleh, M. (2008). Cell death in the host response to infection. Cell Death Differ 15, 1339-1349.

Makino, K., Oshima, K., Kurokawa, K., Yokoyama, K., Uda, T., Tagomori, K., Iijima, Y., Najima, M., Nakano, M. \& other authors
(2003). Genome sequence of Vibrio parahaemolyticus: a pathogenic mechanism distinct from that of $V$ cholerae. Lancet 361, 743-749.

Miller, V. L. \& Mekalanos, J. J. (1988). A novel suicide vector and its use in construction of insertion mutations: osmoregulation of outer membrane proteins and virulence determinants in Vibrio cholerae requires toxR. J Bacteriol 170, 2575-2583.

Milton, D. L., O'Toole, R., Horstedt, P. \& Wolf-Watz, H. (1996). Flagellin A is essential for the virulence of Vibrio anguillarum. J Bacteriol 178, 1310-1319.

Monack, D. M., Mecsas, J., Ghori, N. \& Falkow, S. (1997). Yersinia signals macrophages to undergo apoptosis and YopJ is necessary for this cell death. Proc Natl Acad Sci U S A 94, 10385-10390.

Neyt, C. \& Cornelis, G. R. (1999). Insertion of a Yop translocation pore into the macrophage plasma membrane by Yersinia enterocolitica: requirement for translocators YopB and YopD, but not LcrG. Mol Microbiol 33, 971-981.

Nonaka, T., Kuwabara, T., Mimuro, H., Kuwae, A. \& Imajoh-Ohmi, S. (2003). Shigella-induced necrosis and apoptosis of U937 cells and J774 macrophages. Microbiology 149, 2513-2527.

Ono, T., Park, K. S., Ueta, M., lida, T. \& Honda, T. (2006). Identification of proteins secreted via Vibrio parahaemolyticus type III secretion system 1. Infect Immun 74, 1032-1042.

Park, K. S., Ono, T., Rokuda, M., Jang, M. H., Okada, K., lida, T. \& Honda, T. (2004). Functional characterization of two type III secretion systems of Vibrio parahaemolyticus. Infect Immun 72, 6659-6665.

Samali, A., Zhivotovsky, B., Jones, D., Nagata, S. \& Orrenius, S. (1999). Apoptosis: cell death defined by caspase activation. Cell Death Differ 6, 495-496.

Sganga, G., Cozza, V., Spanu, T., Spada, P. L. \& Fadda, G. (2009). Global climate change and wound care: case study of an off-season Vibrio alginolyticus infection in a healthy man. Ostomy Wound Manage 55, 60-62.

Simon, R., Priefer, U. \& Pühler, A. (1983). A broad host range mobilization system for in vivo genetic engineering: transposon mutagenesis in gram negative bacteria. Nat Biotechnol 1, 784-791.

Thompson, C. C., Vicente, A. C., Souza, R. C., Vasconcelos, A. T., Vesth, T., Alves, N., Jr, Ussery, D. W., lida, T. \& Thompson, F. L. (2009). Genomic taxonomy of vibrios. BMC Evol Biol 9, 258.

Troisfontaines, P. \& Cornelis, G. R. (2005). Type III secretion: more systems than you think. Physiology (Bethesda) 20, 326-339.

Xie, Z. Y., Hu, C. Q., Chen, C., Zhang, L. P. \& Ren, C. H. (2005). Investigation of seven Vibrio virulence genes among Vibrio alginolyticus and Vibrio parahaemolyticus strains from the coastal mariculture systems in Guangdong, China. Lett Appl Microbiol 41, 202-207.

Zhao, Z., Ke, F., Huang, Y. H., Zhao, J. G., Gui, J. F. \& Zhang, Q. Y. (2008). Identification and characterization of a novel envelope protein in Rana grylio virus. J Gen Virol 89, 1866-1872.

Zhao, J. J., Chen, C., Zhang, L. P. \& Hu, C. Q. (2009). Cloning, identification, and characterization of the rpoS-like sigma factor rpoX from Vibrio alginolyticus. J Biomed Biotechnol 2009, 126986.

Zhou, X., Konkel, M. E. \& Call, D. R. (2009). Type III secretion system 1 of Vibrio parahaemolyticus induces oncosis in both epithelial and monocytic cell lines. Microbiology 155, 837-851.

Edited by: D. L. Gally 